\title{
Is Electron Microscopy Relevant Anymore in Diagnosing Disease?
}

\author{
W.T Gunning
}

Department of Pathology, University of Toledo, Toledo, USA

Ultrastructural pathology has lost favor during the past decade for a variety of reasons, the most obvious due to the advent of antibody utilization to identify specific epitopes to characterize disease states, especially in terms of neoplasm diagnosis. The most significant use of electron microscopy (EM) for diagnostic purposes occurred in the 1960's through the 1970's during the very time that the ultrastructural anatomy of the cell was being described. The use of EM in pathology remained strong through the 1990's but a number of pressures began to take hold in the 90's that ultimately caused widespread closure of EM facilities in community tertiary care hospitals and continued into the $21^{\text {st }}$ century with many academic hospitals shutting the doors of their EM facilities. The common criticisms of using EM for diagnostic purposes for tissue samples included "It costs too much." and "It takes too long." and other underlying issues also included a lack of both professional and technical expertise. Immunohistochemical techniques (IHC) have become a mainstay of diagnostic protocols when evaluating tumor differentiation/etiology and immunofluorescence, using direct labeling of trapped immunoglobulins in kidney biopsies, is an essential tool to diagnose renal disease. The question at hand: Is electron microscopy relevant anymore in the practice of pathology?

The rationale to criticize the use of EM in pathology is a fallacy! It is true that microscopes and ancillary equipment are expensive, however these instruments are functional for many more years than most instruments utilized in clinical labs. Electron microscopy techniques have been developed that allow tissue sections to be available within 24 hours or less of sample submission. A "secret" never discussed is that utilization of 3-5 antibodies for diagnosis is more than the cost of EM. In fact, some protocols employ as many as 8-10 different antibodies, dependent upon tumor type, and it is well known that antibody specificity and sensitivity may be questionable in some instances. The utilization of the "brown" stains has been of significant benefit without question, as most hospital laboratories can employ IHC reliably for ensuring a correct diagnosis of many cancers. Is electron microscopy relevant anymore in the practice of pathology? The answer is unequivocally: YES!

The utilization of EM in renal pathology is essential; once waning in use for early transplant rejection, it is now very important to aid in diagnosis of humoral rejection by evaluating interstitial capillary basement membranes for evidence of structural alteration. Its use in routine work-up of patients presenting with nephrosis or nephritis remains essential for accurate diagnosis of renal disease. Muscle disease presents a variety of conditions that can be readily diagnosed using ultrastructural evaluation. Clinical history must guide interpretation of muscle biopsies and EM may be essential to correctly diagnose inclusion body myositis from polymyositis for which treatment differs. Metabolic disorders also present a challenge and EM serves a role to guide potential molecular investigations to determine genetic mutations. Bleeding diatheses may be extremely difficult to diagnose without the use of EM. 
Yes, electron microscopy is still relevant in the practice of pathology; it isn't utilized as often as in years past but in a number of situations, a diagnostic dilemma may be easily resolved by employing ultrastructural evaluation.

This presentation will describe some cases of the "wrong diagnosis" and demonstrate ultrastructural features that "saved the day" to make an accurate interpretation of disease. Examples of ultrastructural pathology will also be discussed to underscore the relevance of EM in the practice of pathology.

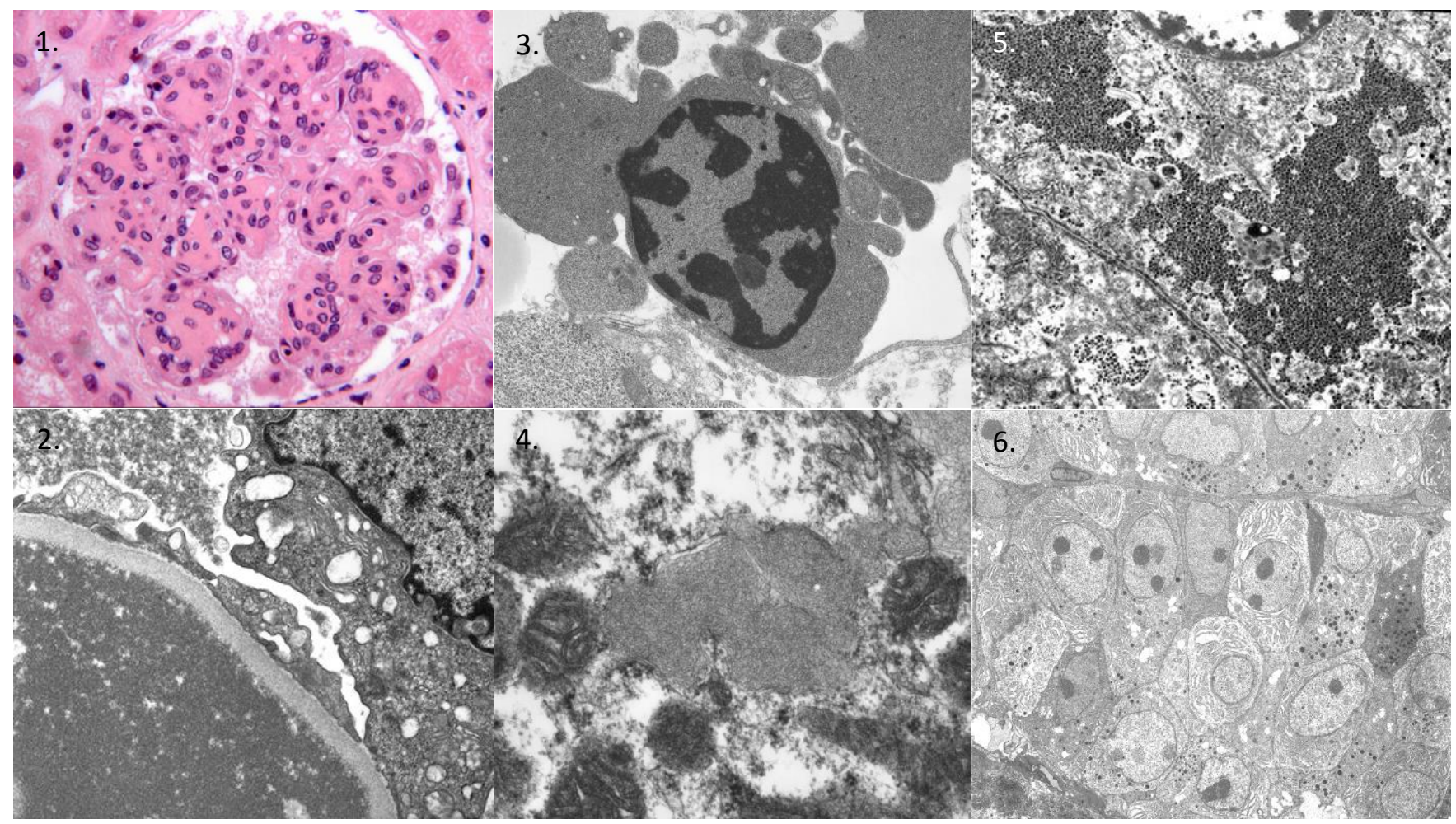

Figure 1. Glomerulus from a diabetic patient with light microscopy features that were misdiagnosed as diabetic nodular sclerosis (K-W diabetic nephropathy).

Figure 2. Ultrastructural morphology of a glomerulus as seen in Fig. 1, demonstrated significant accumulations of immunotactoids; the diabetic patient had a monoclonal gammopathy.

Figure 3. Nucleated red blood cell from a bone marrow biopsy is characteristic of congenital erythropoeitic anemia, Type 1 (CDA-1).

Figure 4. Branched glycogen accumulation in cardiac tissue, consistent with a glycogen storage disease.

Figure 5. Accumulation of glycogen in hepatocytes representing a different glycogen storage disease.

Figure 6. This image was used to make a diagnosis of pancreatic carcinoma whereas the light microscopic diagnosis, utilizing a number of antibodies reactions against the tumor cells, was misdiagnosed as a primary peritoneal carcinoma arising from the ovary. 